\title{
An Effective Hybrid GACSA -based Multi-user Detection for Ultra-WideBand Communications Systems
}

\author{
Jyh-Horng Wen \\ Department of Electrical Engineering, \\ Tunghai University \\ No. 181, Sec. 3, Taichung Harbor Rd., \\ Taichung, Taiwan, R.O.C. \\ e-mail :jhwen@thu.edu.tw
}

\author{
Ho-Lung Hung, Chien-Chi Chao \\ Department of Electrical Engineering, \\ Chienkuo Technology University, \\ Changhua, Taiwan. \\ e-mail: hlh@cc.ctu.edu.tw
}

\author{
Chia-Hsin Cheng \\ Department of Electrical Engineering, \\ National Formosa University \\ No. 64, Wunhua Rd., Huwie, Yunlin \\ 632, Taiwan, \\ e-mail:chcheng@nfu.edu.tw
}

\begin{abstract}
In this paper, we investigate the performance of various interference cancellation techniques in directsequence ultra-wideband (DS-UWB) communication systems. Multiple access interference (MAI) causes the performance of the conventional single user detector in DS-UWB systems to degrade. Due to high complexity of the optimum multiuser detector, suboptimal multiuser detectors with less complexity and reasonable performance have received considerable attention. A hybrid approach that employs a genetic algorithm (GA) and chaos algorithm (CSA) for the MUD problem in UWB communication systems is proposed. By taking advantage of heuristic values and the collective intelligence of GACSA, the proposed detector offers almost the same bit error rate (BER) performance as the full-search-based optimum multiuser detector does, while greatly reducing the computational complexity. The near-far resistance of the GACSA-based multiuser detector is also examined. The good behavior of the proposed approach is demonstrated by means of comparisons in term of bit error rate (BER) performance and implementation complexity with the classical Rake receiver and different multiuser receivers previously proposed in the literature on this subject.
\end{abstract}

Keywords-Ultra-wideband, genetic algorithm, chaos alhorithm, multiuser detection

\section{INTRODUCTION}

Ultra- wideband (UWB) technology is currently being investigated as a promising solution for short range, highcapacity wireless communications systems. Unlike conventional radio systems, UWB systems have a number of advantages that make them attractive for consumer communication applications, such as low cost, low power consumption, low complexity, and high data rate transmission, etc [1-2]. To realize the multiple accesses technique in UWB systems, two commonly used approaches is time hopping (TH) and direct sequence (DS) techniques [3-4]. When using DS technique, pseudo-random code is applied to spread the data bit into multiple chips, just as in conventional DS code division multiple assess (DS-CDMA) systems, and the users are separated by independent spread. In the DS-UWB communication system, multiple access interference (MAI) is the main source of interference. Additionally, it is well known that MAI limits DS-UWB system capacity. Multiuser detection (MUD) is a powerful technique to combat MAI and to improve the performance of UWB systems. Considering the large complexity involved in optimal multiuser detection (OMUD), which is exponential in the number of active users, most of the current work is centered around investigating suboptimal approaches.

Some sub-optimal approaches, such as differential receive and adaptive receivers were proposed [5-6]. These techniques do not require channel estimation and allow capturing a large amount of the received energy. To reduce the complexity of this optimum detector various suboptimum multiuser have been developed in the past several years [4], [5-12]. For examples, the minimum-meansquared error (MMSE) MUD has been described in [5], while an interference cancellation (IC) based MUD has been proposed in [6] [9]. The traditional receiver for such a UWB system is a simple matched filter [1-2] and the performance is degraded due to the MAI and ISI. Moreover, using intelligent computation techniques seems to be a feasible approach to achieve a bit error rate (BER) performance close to that of the OMUD when reducing computational complexity. Hybrids MUD have been proposed [7-8] as have other distinct approaches [9]. While each has its own merits and drawbacks, we will focus on a multistage approach which performs parallel interference cancellation (PIC) at each stage.

In resent years, there are many new intelligence MUD techniques which utilize some genetic algorithms (GA) [1014], particle swarm optimization (PSO) [15], and neural networks (NN) [16] have lower computational complexity. In all kinds of techniques, the evolutionary computation algorithm has proven to be an effective way to design the sub-optimum multiuser detectors. In [9-10], it is shown that GA based MUD approaches the single-user performance bound at a lower complexity as compared with optimal 
maximum likelihood (ML) optimum detector. GA can get the optimum solution for multidimensional engineering problems. Furthermore, GA algorithm has suffered from some deficiencies. It is well known that premature convergence degrades the performance of GA and reduces the search ability [11]. In addition, a change in the genetic population through generation results in the destruction of previous knowledge of the problem [12].

The GA is a stochastic search algorithm whose procedures are based on the Darwinian models of natural selection and evolution [4]. Given some arbitrary initial solutions, the GA will generate the better solution through a series of genetic operations including selection, crossover, and mutation. Furthermore, the GA searches the solution space in parallel, that is, a set of possible solutions are manipulated in the same generation, so multiple local optimum can be reached simultaneously and thereby the likelihood of finding the global optimum is increased. The genetic algorithm based multiuser detector (GA-MUD) [1113 is one of the sub-optimum multiuser detectors that evolves from the OMUD by replacing the exhaustive search scheme with the GA [11], and has attracted much attention in recent years. By means of GA's powerful search ability, the GA-MUD can attain sub-optimum performance with less complexity compared to the OMUD [12]. One of the proposed GA-MUDs uses the GA to provide a good initial point for the successive stage of the multistage detectors. A modified GA that adjusts the operations of the GA to improve the BER performance is proposed in [13]. However, the performance of the GA-MUD still can not approach a sub-optimal, which is far from the single user bound, at high system load. Thus, after a low-complexity GA detector, the parallel interference cancellation (PIC) scheme [10] is used to simultaneously subtract the interference from each user's received signal. However, at heavy system load, the multistage conventional PIC (CPIC) approach suffers performance degradation due to a poor cancellation, which is brought about by the relatively high error rate of bit decisions in the preceding stage [12]. Thus, the partial cancellation contrarily is a better policy than the complete cancellation [13]. Consequently, in this paper, we proposed a low-complexity iterative MUD approach for DS-UWB communication systems which can effectively alleviate the harmful effects of MAI. The proposed method is based upon the use of iterative processing techniques, which have already been successfully applied to much wireless communication system and, in particular, to the MUD case. Using this approach, the CSA is embedded into the GA to improve further the fitness of the population at each generation. Such a hybridization of the GA the CSA reduces its computation complexity by providing faster convergence.

The remainder of this correspondence is organized as follows. Section II describes the signal model of the DSUWB system and Section III introduces the proposed particle swarm optimization technique, and multiuser detection based on the GA-CSA technique. Section IV, simulation results are provided to demonstrate the performance of the proposed detector. Conclusion and discussion are given in Section V.

\section{SYETEM AND CHANNEL MODEL}

\section{A. Transmitter Model}

In this section describes a simple model for a DS-UWB communication system employing multiuser detector which will be employed for the proposed of analysis in this paper. We assume a $K$-users DS-UWB system over the UWB indoor multipath fading channels, where each user employs unique DS spreading code. The transmitted signal $q_{k}(t)$ for the $k$ th user is obtained by spreading a set of binary phaseshift keying (BPSK) data symbol $\left\{b_{k}[i]\right\}$ onto a spreading waveform $s_{k}(t)$, which is written as follows:

$q_{k}(t)=\sqrt{E_{k}} \sum_{i=1}^{P} b_{k}[i] s_{k}\left(t-i T_{b}\right)$,

where $E_{k}$ is the symbol energy of the $k$ th user, $P$ is the packet size, $b_{k}[i] \in\{ \pm 1\}$ is the $i$ th data symbol of the $k$ th user, and $T_{b}$ is the symbol interval duration. The spreading waveform $s_{k}(t)$ is also written as follows:

$s_{k}(t)=\frac{1}{\sqrt{G}} \sum_{n=0}^{N_{c}-1} c_{k, n} w\left(t-n T_{c}\right)$,

where $G=\sum_{n=1}^{N_{c}} c_{k, n}^{2}, k=1,2, \ldots, K, c_{k, n} \in\{ \pm 1\}$ is the $n$th chip of the $k$ th user, $N_{c}$ is the chip numbers, $T_{c}$ is the chip interval duration, and $w(t)$ is the chip waveform of duration $T_{c}=T_{b} / N_{c}$.

\section{B. Multipath Channel Model}

In this paper, we use the IEEE 802.15.3a indoor channel model, which is based on a modified Saleh-Valenzuela ( $\mathrm{S}-\mathrm{V})$ model where multipath components arrive in clusters, each of which could contain several components namely rays [17]. For the UWB indoor transmission environment, the channel impulse response of UWB indoor channel model is modeled as

$h_{k}(t)=\sum_{l=1}^{L_{k}} \alpha_{k, l} \delta\left(t-\tau_{k, l}\right)$
$=\sum_{l=1}^{L_{k}} \alpha_{k, l} \delta\left(t-(l-1) T_{c}\right)$,

where $L_{k}$ denotes the total number of propagation paths of the $k$ th user, $\alpha_{k, l}$ is the channel coefficient of the $l$ th path of the $k$ th user and $\tau_{k, l}$ is the multipath delay of the $l$ th path of the $k$ th user. In this thesis, we suppose that the multipath delay $\tau_{k, l}$ is an integral multiple of $T_{c}, L_{1}=L_{2}=\ldots=L_{K}=$ $L$, and the system is assumed to be synchronous. In system performance analysis, the commonly adopted UWB 
multipath channel models are those standard statistical models established by IEEE 802,15.3a task group, i.e., CM1 CM4. These standard models are based on modified $\mathrm{S}-\mathrm{V}$ model, of which the clustered multipath components obey a lognormal amplitude distribution and an equiprobable polarity distribution.

\section{Review of Multiuser Detectors in DS-UWB communication Systems}

This section describes the construction of the conventional multiuser detector and optimum MUD for UWB communication systems. When passing the signal through the indoor environment, the obstacles in the transmitted path will cause the multipath transmission. Therefore, the total received signal can be formulated as follows:

$$
\begin{aligned}
& r(t)=\sum_{k=1}^{K} q_{k}(t) \otimes h_{k}(t)+n(t) \\
& =\sum_{k=1}^{K} \sqrt{E_{k}} \sum_{i=1}^{P} b_{k}[i] v_{k}\left(t-i T_{b}\right)+n(t),
\end{aligned}
$$

where $\otimes$ is linear convolution, $n(t)$ is zero-mean additive white Gaussian noise and $v_{k}(t)=s_{k}(t) \otimes h_{k}(t)$ is defined as template signal of the $k$ th user, which is a convolution between the $k$ th user's spreading code and channel coefficient.

\section{Conventional Detector}

The template signal $v_{k}(t)$ that is transmitted over a channel is corrupted by channel noise. Hence, the function of the receiver must detect the template signal $v_{k}(t)$ for each user. According to [5], we note that a filter which is matched to a template signal $v_{k}(t)$ of duration $\left(N_{c}+L-1\right) T_{\mathrm{c}}$ is characterized by an impulse response. The channel response for $k$ th user can be written as follows:

$$
h_{\text {opt }, k}(t)=v_{k}^{*}(-t) \text {. }
$$

So, the output of the filter which is matched to a template signal $v_{k}(t)$ can be written as follows:

$$
\begin{aligned}
& y_{k}(t)=r(t) \otimes h_{o p t, k}(t) \\
& =r(t) \otimes v_{k}^{*}(-t) \\
& =\sum_{m=1}^{K} \sqrt{E_{m}} \sum_{i=1}^{P} b_{m}[i] v_{m}\left(t-i T_{b}\right) \otimes v_{k}^{*}(-t)+n(t) \otimes v_{k}^{*}(-t),
\end{aligned}
$$

and the discrete-time impulse response sampling at $t=i T_{b}$ is represented as follows:

$$
y_{k}[i]=y_{k}\left(i T_{b}\right) \text {. }
$$

Then the discrete-time received signal after sampling $\left(i T_{b}\right)$ is written as follows:

$$
y_{k}[i]=\sum_{m=1}^{K} \sqrt{E_{m}} \sum_{j=1}^{P} b_{m}[j] v_{m}[i-j] \otimes v_{k}^{*}[-i]+n[i] \otimes v_{k}^{*}[-i]
$$

$$
\begin{aligned}
& =\sum_{m=1}^{K} \sqrt{E_{m}} \sum_{j=1}^{P} b_{m}[j] R_{m, k}[i, j]+\tilde{n}_{k}[i] \\
& =\underbrace{\sqrt{E_{k}} b_{k}[i] R_{k, k}[i, i]}_{\text {desired signal }}+\underbrace{\sqrt{E_{k}} \sum_{\substack{j=1 \\
j \neq i}}^{P} b_{k}[i] R_{k, k}[i, j]}_{\text {ISI }}
\end{aligned}
$$$$
+\underbrace{\sum_{\substack{m=1 \\ m \neq k}}^{K} \sqrt{E_{m}} \sum_{j=1}^{P} b_{m}[j] R_{m, k}[i, j]}_{\text {MAI }}+\tilde{n}_{k}[i],
$$

where

$$
\begin{aligned}
& R_{m, k}[i, j]=v_{m}[i-j] \otimes v_{k}^{*}[-i], \\
& \tilde{n}_{k}[i]=n[i] \otimes v_{k}^{*}[-i] .
\end{aligned}
$$

Hence, the signal that received by a CD can be detected:

$$
\hat{b}_{k}^{C D}[i]=\operatorname{sgn}\left\{y_{k}[i]\right\},
$$

where a CD structure is shown in Fig. 1.

\section{E. Maximum Likelihood Detector}

The optimum MUD performs maximum-likelihood sequence detection jointly across all users' sequences [5-6]. According to [4-6], the optimal multiuser detector can be achieved by maximum a posteriori (MAP) estimation. Because the probability of $b_{k}[i]=+1$ is equal to the that of $b_{k}[i]=-1$, the maximum likelihood (ML) estimation can be generalized by the MAP estimation. As a result, the optimal multiuser detector that fulfils ML sequence estimation [5] gives the best performance. However, its computational complexity which grows exponentially with the number of the users forbids application in real system. The search for $\mathbf{b}$ is a combinatorial optimization problem detailed in [5] whose complexity grows exponentially with $K$. Though impractical given large $K$, its performance establishes a benchmark for multiuser design. In UWB systems, the number of zero cross-correlation entries in $\boldsymbol{R}$ can very be large and can help decouple the ML sequence detection problem into much smaller, independent ML sequence detection problems.

$\hat{\mathbf{b}}^{\mathrm{ML}}=\arg \max _{\mathbf{b} \in[-1,+1]^{K P}}\left[2 \mathbf{b}^{T} \mathbf{A y}-\mathbf{b}^{T}(\mathbf{A R A}) \mathbf{b}\right]$.

\section{GENETIC ALGORITHM AND ChaOs Algorithm}

\section{Solution Representation}

For the subsequent genetic operations, the trial solution to the addressed problem must be encoded into the string form first. An encoded solution is referred to as a chromosome and its elements are referred to as the genes. The multiuser detection can be regarded as an optimization problem that finds the most likely combination of the binary transmitted bits $\hat{\mathbf{b}}_{\mathrm{OMD}, p}$. Since the configuration of the trial solution $\left[\hat{b}_{1, p}, \hat{b}_{2, p}, \cdots, \hat{b}_{K, p}\right]$ is already an antipodal binary string of 
length $K$, the encoding process is unnecessary.

\section{Initialization}

For each time the GA being carried out, a chromosome set with $P_{c}$ members named the chromosome population is created in order to produce the better solutions by applying the genetic operations, where $P_{c}$ is known as the population size. Generally, the larger the population size, the faster the convergence rate but the higher the computational complexity. In this paper, the seed chromosome in the initial population is created by the Rake receicer. The Rake receiver first makes a rough decision $\hat{\mathbf{b}}_{p}=\left[\hat{b}_{1, p}, \hat{b}_{2, p}, \cdots, \hat{b}_{K, p}\right]$ according to the received equivalent baseband signal $r(i)$ in (10) and then passes its output to the GA for further processes.

\section{Cost Evaluation}

In the cost evaluation process the GA employs the problem-dependent objective function to evaluate the cost or fitness of a solution, which represents how closely the chromosome fits the addressed problem. A high fitness or low cost reflects the excellence of the chromosome. The objective of our system is to find the $\hat{\mathbf{b}}$ that has the minimum cost. Consequently, we define the cost function of a chromosome for the $i$ th bit duration as

$$
\cdot \hat{\mathbf{b}}^{\mathrm{ML}}=\arg \max _{\mathbf{b} \in[-1,+1]^{K P}}\left[2 \mathbf{b}^{T} \mathbf{A y}-\mathbf{b}^{T}(\mathbf{A R A}) \mathbf{b}\right] \text {. }
$$

\section{Selection}

Selection is the operation that chooses the chromosomes from the parent population to constitute a selected population for crossover. Since the parent chromosomes with advantageous genes are more likely to produce the better offspring chromosomes, only certain parent chromosomes will have the chance to produce the offspring chromosomes. The selected population consists of $P_{c}$ chromosomes chosen from the parent population with probabilities that are inversely proportional to their costs by using the roulette wheel selection scheme [11]. Then, the selection rate of the $k$ th chromosome is

$$
P_{S, k}=\frac{C\left(\hat{\mathbf{b}}_{k}\right)^{-1}}{\sum_{j=1}^{P_{c}} C\left(\hat{\mathbf{b}}_{j}\right)^{-1}},
$$

where $C\left(\hat{\mathbf{b}}_{j}\right)$ is the cost of the $j$ th chromosome.

\section{Crossover}

The crossover is the operation that exchanges the genes of two chromosomes to generate a new pair of offspring chromosomes. Through crossover, the offspring chromosomes are expected to be superior to their producer because they inherit the merits of both parents. Three commonly used crossover schemes are one-point, multipoint, and uniform crossover [4]. The simplest crossover scheme, the one-point crossover, is utilized in this study. The probability that the crossover operation is applied to the selected chromosomes, which is known as the crossover rate, is set to 1 .

\section{Offspring Mutation}

The mutation is the operation that randomly alters the genes of the crossover results for increasing the diversity of genes. Sometimes the members of the selected population are not diverse enough to find any better solution even if the global optimum is not reached yet. This situation is known as the premature convergence and may happen when one of the parents has a relatively low cost compared to the others', in which this outstanding chromosome predominates the selected population, resulting in a considerable amount of identical offspring chromosomes. Therefore, the mutation operation is introduced to prevent the premature convergence. The GA with a high mutation rate $P_{m}$ is more likely to escape from the local optimum but has the slower convergence. Conventionally, the mutation rate $P_{m}$ is usually smaller than the crossover rate. In this paper, the $P_{m}$ is set by 0.1 to compromise between the optimization and convergence.

\section{Elitist Replacement}

The mutation and crossover are the operations that modify genes within chromosomes. To avoid destroying the good solutions during the mutation and crossover, we replace a small portion of offspring population with the good chromosomes in the parent population. This is called the elitist replacement. In this paper, the elitist replacement is implemented by selecting the best $P_{c}$ chromosomes from the combination of the parent and offspring population [11].

\section{Iteration}

Unlike the other sub-optimum multiuser detectors, the GA is an iteration scheme. We can repeat the procedures mentioned in parts $3 \sim 7$ to refine our solution. In GA, each iteration is called a generation. It is known that when the pre-defined generation number $G_{n}$ is reached the iteration will be terminated and the best chromosome in the last generation will be taken as the result of the detection. With the aid of the elitist replacement, the best chromosome of the offspring population is never worse than that of the parent population. This ensures the discovery of better solution after certain generations.

The standard adaptive genetic algorithm (AGA) is proposed by Srinvas [11]. its main idea is that when the fitness values of population tend to convergence, the probability of the occurrence of the genetic operators will be increased so as to avoid the premature convergence, where when the fitness values of population tend to divergence, the probability of the occurrence of the genetic operator will be decreased so as to converge to the optimum. Figure 1 the flow chart of the GA-based detection scheme for DS-UWB systems. 
The probabilities of crossover and mutation are defined as follows:

$$
\begin{aligned}
& p_{c}=\left\{\begin{array}{l}
e_{1} \frac{f_{\max }-f^{\prime}}{f_{\max }-f_{\text {avge }}}, f^{\prime} \geq f_{\text {avge }} \\
e_{3}, f^{\prime}<f_{\text {avge }}
\end{array}\right\} \\
& p_{m}=\left\{\begin{array}{l}
e_{2} \frac{f_{\max }-f}{f_{\text {max }}-f_{\text {avge }}}, f \geq f_{\text {avge }} \\
e_{4}, f<f_{\text {avge }}
\end{array}\right\}
\end{aligned}
$$

where $f_{\max }$ and $f_{\text {avge }}$ are the maximal fitness value and average fitness value of the population, respectively. $f^{\prime}$ is the larger one of the fitness values of two individuals to be crossed, and $f$ it is the fitness value of the individual to be mutated. $e_{1}, e_{2}, e_{3}$ and $e_{4}$ are constants predetermined in the range $[0,1]$.

The basic idea of AGA is that, during the genetic search process, when the individual fitness value is less than the average fitness values of the population, larger rates of crossover and mutation operators should be adopted, and vice versa. This scheme can prevent the genetic search process from prematurely converging to local optimal solutions by regulating the balance between exploration and exploitation in the solution space. The procedure of AGA can be depicted as follows:

Step 1: Initialize the population and various parameters.

Step 2: Calculate the fitness values including individual fitness values and average fitness values of the population.

Step 3: Compute the crossover and mutation rates according to formulas (13) and (14).

Step 4: execute genetic operation including selection, crossover and mutation.

Step 5: when the current generations is less than the maximal generation, turn to step 2; otherwise, terminate the program and return the optimal searching. Adaptive Genetic algorithm combined with chaos searching [17] (GACSA):

The proposed approach GACSA is developed on the basis of the standard GA by introducing chaos searching and the other set of crossover and mutation rates so as to guide to the whole population to evolve in the solution space. In GACSA, the chaos searching algorithm is used to local exploration for obtaining the local optimum while the AGA with two sets of crossover and mutation rates is responsible for global exploitation. In order to fulfill chaos searching, here, a premature decision identifier $\eta$ is employed: assume that $\bar{f}^{i}$ is the average fitness values at generation $i$, which is computed by $\frac{1}{P} \sum_{n=1}^{P} f_{n}^{i}$, where $f_{n}^{i}$ represents the fitness value of the $n$th individual in the $i$ th generation, $P$ is the population size. At the same time, suppose the best individual's fitness is $f_{\max }^{i}, \bar{f}^{\prime}$ is the average of all the individuals whose fitness values are greater than $\bar{f}^{i}$. And then let $\eta=f_{\max }^{i}-\bar{f}^{\prime}$. It is known that the rates of crossover and mutation operators have a significant effect on the convergence of GA during the search process. Thus, the other set of crossover and mutation rates adopted in this paper is devised with $\eta$ as follows:

$$
\begin{aligned}
& p_{c}=1+0.3\left[-\frac{1}{1+\exp \left(-e_{1} \eta\right)}\right] \\
& p_{\text {mut }}=0.03+0.03\left[-\frac{1}{1+\exp \left(-e_{2} \eta\right)}\right]
\end{aligned}
$$

where $e_{1}$ and $e_{2}$ are the positive numbers predetermined. The detailed procedure of GACSA is described as follows: Step 1: Initialize the population and various parameters.

Step 2: Calculate the fitness values of each individual and the premature decision identifier $\eta$.

Step 3: When $\eta$ is greater or equal to $\eta^{*}$ and current generation (GEN) is greater than maximal generation (M), $\mathrm{M} / 2$, carry out the following steps for chaos searching.

1). Let $d=1$.

2). Execute chaos search from step 2 to 5 as shown in procedure chaos optimization algorithm, and the rest (D-1) individuals keep invariant.

3). $d \rightarrow d+1$. If $\mathrm{d}$ reaches $\mathrm{D}$, terminate the chaos search; otherwise, turn to 2) to optimize the next variable. When $\eta$ is greater or equal to $\eta^{*}$ and GEN is less or equal to $\mathrm{M} / 2$, calculate the rates of crossover and mutation operators by formulas 4) and 5), and carry out the corresponding genetic operations. Otherwise, turn to Step 4.

Step 4: Calculate the crossover and mutation rates according to equations (13) and (14).

Step 5: Execute genetic operations including selection, crossover and mutation.

Step 6: When the GEN is smaller than the maximal generation $(\mathrm{M})$, turn to Step 2; otherwise, terminate the program and return the optimal solutions.

\section{Simulation RESUlts}

In this section, the simulations of multiuser transmission for DS-UWB radio systems under the modified S-V channel that GACSA based MUD algorithm is adopted are shown in Figs. 2-5. The UWB CM 1-4 which are discussed in paper indicate the different transmission distance for indoor environment, and all Rake receivers is adopted for CM 1-4. We assume that the packet size is 4 bits and the number of users is 10 on DS-UWB systems 


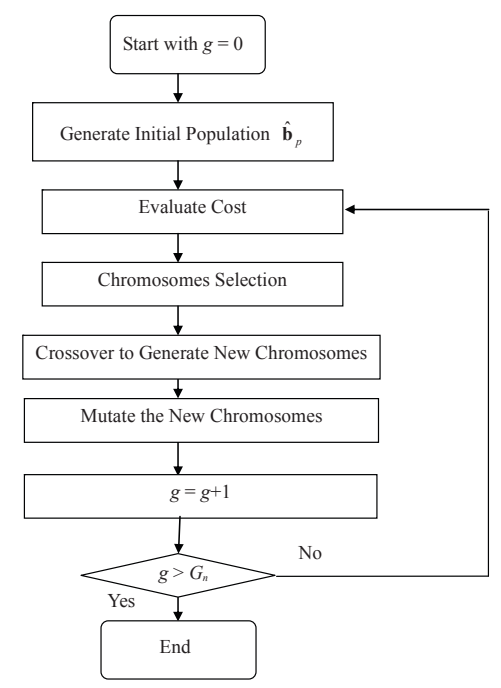

Fig. 1. The flow chart of the GA-based detection scheme for DSUWB systems.

. Fig. 2 shows the performance of GACSA based MUD, the optimum detector, and several suboptimum detectors, for $K=10$. The performance of HNN detector for DS-UWB systems is better with the increase of iteration. However, the performance improvement is no longer obvious when it achieves about 100 iterations. That is because the HNN detector has many local minimum, but it is unable to determine which minimum is the global minimum. Since the information of $\mathbf{R}$ is known for HNN detector, the performance of HNN detector always is better than CD when it achieves about 50 iterations. Unfortunately, the performance of HNN detector is poorer than original GA, original PSO, GACSA and OMUD detectors for DS-UWB in CM 1. On the other hand, as for the BER of the original GA-based MUD, an error floor is observed foe the results show in the figure. This is because of the limitations of the GA associated with the particular set of individual and generation values. In contrast, the BER of the proposed GACSA based MUD demonstrated a perfect approach similar to that of OMUD with less computational complexity compared with that of the original PSO- and GA-based MUD.

Further, in Figs.3-5 the performance of GACSA based detector is depicted for CM 2, CM 3 and CM 4, respectively. The performance of $\mathrm{HNN}$ detector is approximated other suboptimum detectors at $\mathrm{SNR}=0-6 \mathrm{~dB}$ for other $\mathrm{DS}-\mathrm{UWB}$ channel models. But, its performance is worse at $\mathrm{SNR}=8$ $12 \mathrm{~dB}$ for others. The neuron output of HNN detector with sign activation is either +1 or -1 , but the neuron output of HNNdetector is distributed form -1 to +1 . As can be observed in Figs. 2-4, the performance of our algorithm is better than that of all existing suboptimum schemes with same level of complexity. Moreover, the performance of GACSA detector is approximated OMUD at SNR $=6-12 \mathrm{~dB}$ for other UWB channel models.

\section{CONCLUSION}

To reduce computational complexity of the optimal multi-user detector, a novel hybrid algorithm that employs GA and CSA is present. In this paper, we proposed a new suboptimum multiuser detector for DS-UWB systems, which utilizes a hybrid algorithm to decide on the transmitted bits. Using this approach, the CSA is embedded into GA to improve further the fitness of the population at each generation. Such a hybridization of the GA with the CSA based MUD reduces its computational complexity by providing faster convergence. The complexity of the proposed is approximately $O\left(K S_{T}\left(P_{p}+P_{i}\right)\right)$, and it is evident that its performance is significantly better and more robust compared to other examined suboptimum schemes. Simulations results are provide to show that the proposed detector has significant performance improvement over the detectors based on HNN, PSO and CD in term of MAI.

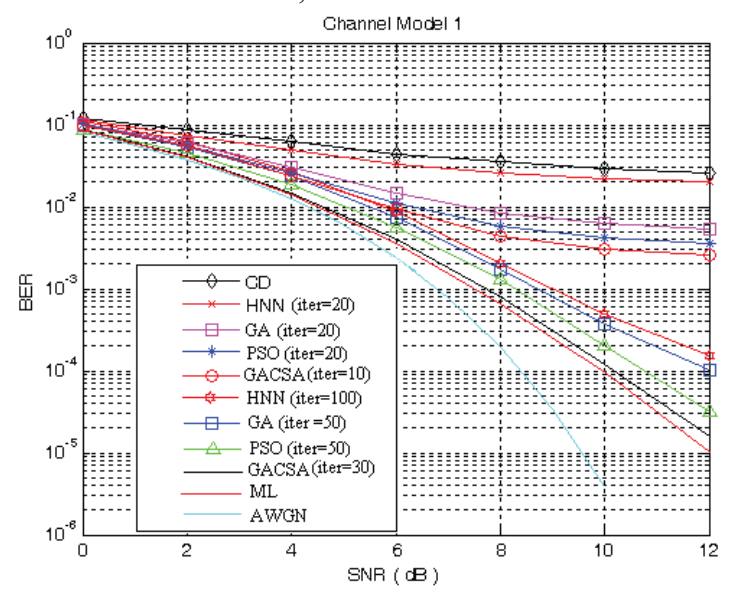

Fig.. 2 The simulation of BER for DS-UWB systems that employs GACSA, $\mathrm{CD}, \mathrm{GA}, \mathrm{PSO}, \mathrm{ML}$ and $\mathrm{HNN}$ detectors with UWB CM 1 when $\mathrm{K}=10$

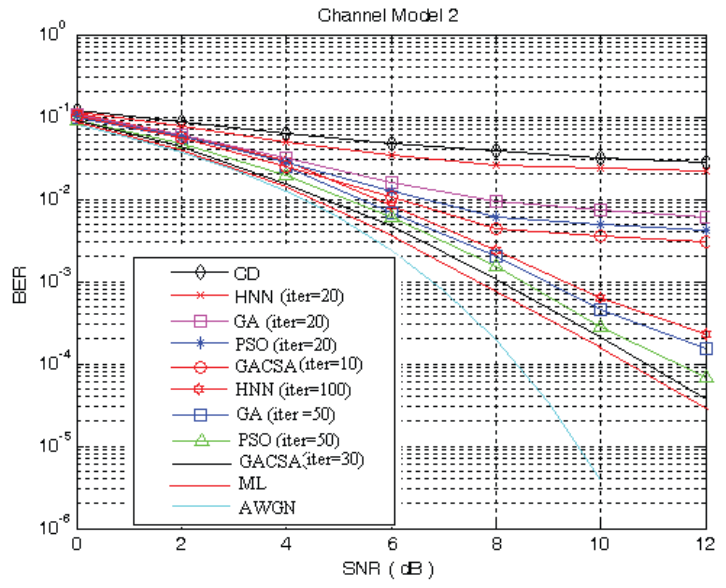

Fig. 3 The simulation of BER for DS-UWB systems that employs GACSA, $\mathrm{CD}, \mathrm{GA}, \mathrm{PSO}, \mathrm{ML}$ and $\mathrm{HNN}$ detectors with UWB CM 2 


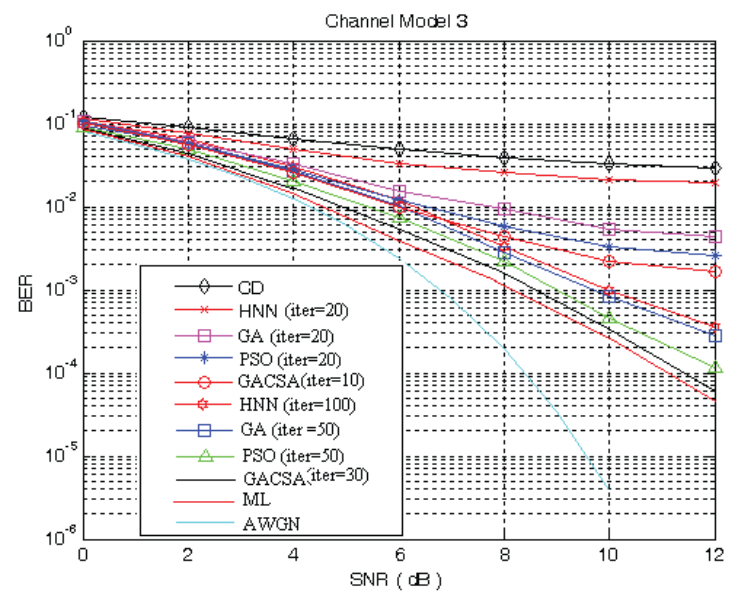

Fig. 4 The simulation of BER for DS-UWB systems that employs GACSA, $\mathrm{CD}, \mathrm{GA}, \mathrm{PSO}, \mathrm{ML}$ and HNN detectors with UWB CM 3

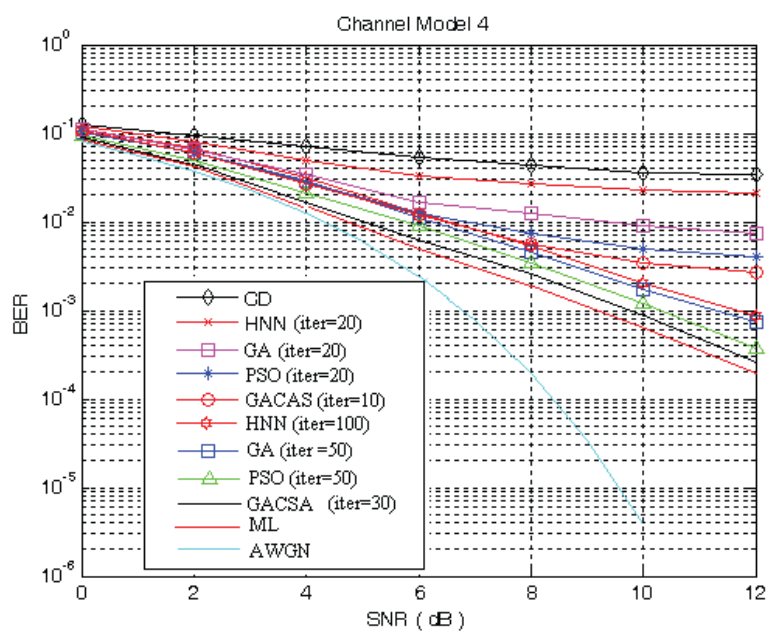

Fig. 5 The simulation of BER for DS-UWB systems that employs GACSA, CD, GA, PSO,ML and HNN detectors with UWB CM 4

\section{REFERENCES}

[1]. Win M Z, Scholtz RA. Impulse radio: How it works. IEEE Commun. Lett; vol.2, pp. 36-38, 1998.

[2]. Linging Yang, Georgios B. Giannakis. Ultra-Wideband communications an idea whose time has come. IEEE Signal Processing Magazine ; pp.26-54, 2004.

[3]. Ismail Guvenc, Huseyin Arslan. A review on multiple access interference cancellation and avoidance for IR-UWB. ELSEVIER Singal Processing ;vol.87, pp.623-563, 2007.
[4]. Verdú S. Multiuser Detection. Cambridge, U.K.: Cambridge Univ. Press, 1998.

[5]. Li Q, Rusch L. A. Multiuser detection for DS-CDMA UWB in the home environment, IEEE J. Select Areas Commun. vol.20, pp.1701-1711. Dece.2002.

[6]. Nejib Boubaker and Khaled Ben Letaief, Combined multiuser successive interference cancellation and partial rake reception for Ultra-wideband wireless communication, 2004, pp. 1209-1212.

[7]. J. R. Foerster, The performance of a direct-sequence spread ultra wide-band system in the preseence of multipath, narrowband interference, and multiuser interference, IEEE Conf. Ultra wideband Syst. Technologies, pp. 87-91, May 2002.

[8]. Yoon YC, Kohno R. Optimum multiuser detection in Ultra wide-band multiple-access communication systems, In Proc IEEE International confer on Commun. New York City, NY, pp.812-816, 2002.

[9]. Lili Lin and Anding Wang, New hybrid multiuser receiver for DS-UWB system, IEEE Wireless Communications, Networking and Mobile Computing, WiCOM , pp.1-5, Oct. 2008.

[10]. Tan-Hsu Tan, Yung-Fa Huang, Chung-Weng Lin, and RayHsiang Fu, Performance improvement of multiuser detection using a genetic algorithm in DS-CDMA UWB systems over an extreme NLOS multipath channel. In Proc. of IEEE SMC 2006, vol. 3, pp. 1945-1950, Oct. 2006.

[11]. D. B. Fogle, Evolutionary Computation: Toward a New Philosophy of Machine Intelligence, $2^{\text {nd }}$ ed. Piscatewy, NJ: IEEE Press, 2000.

[12]. C. Ergun and K. Hacioglu, "Multiuser detection using a genetic algorithm in CDMA communication systems," IEEE Trans. Commun., vol. 48, no. 8, pp. 1374-1383, Aug. 2000.

[13]. K. Yen and L. Hanzo, "Genetic-algorithm-assisted multiuser detection in asynchronous CDMA communications," IEEE Trans. Veh. Technol., vol. 53, Issue 5, pp. 1413 - 1422, Sept. 2004.

[14]. S. Abedi and R. Tafazolli, "Genetically modified multiuser detection for code division multiple access systems," IEEE $J$. Select. Areas in Commun., vol. 20, pp. 463-473, Feb. 2002.

[15]. H.-L. Hung, and J.-H. Wen, An Adaptive Multistage Multiuser Detector for MC-CDMA Communication Systems Using Evolutionary Computation Technique," to appear in Springer: Wireless Personal Communications, 2009.

[16]. Sitao Wu and Tommy W, S. Chow, Self-Organizing and self-evolving neurons: a new neural network for optimization, IEEE Trans. on Neural Networks, vol. 18, pp. 385-396, March 2007.

[17]. Silva C. P. Survey of Chaos and its applications. Proceeding of the 1996 IEEE MTT-S Internation microwave symposium digest, San Francisco, USA, pp. 1871-1874.

[18]. Saleh AA, Valenzuela RA. A statical model for indoor multipath propagation. IEEE J. Select. Areas Commun.; vol.5, pp. 128-137, 1987. 